\title{
Historia de la contaminación de la bahía de Ite por la empresa minera Southern Perú
}

\author{
History of the pollution in Ite bay by the mining company Southern \\ Peru
}

\author{
${ }^{1}$ Alexander Churata Neira \\ ${ }^{2}$ Wilberth Abraham Chambilla Ccosi
}

ORCID: 0000-0001-9288-9078

ORCID: 0000-0001-9677-6302

\section{RESUMEN}

El presente artículo tuvo como objetivo relatar la historia de la contaminación de la bahía de Ite, ubicada en Tacna, como un caso importante de contaminación por incremento de sólidos y la presencia de metales pesados por la actividad de las minas de Toquepala y Cuajone. Iniciando sus operaciones entre 1960 y 1976, los humedales de Ite se han visto expuestos a una gran contaminación por los relaves depositados, deteriorando el litoral marino y modificándolo hasta alcanzar un área total de más de 1600 hectáreas, con un kilómetro y medio de ancho y 12 kilómetros de largo. En diciembre de 1996, la descarga de relaves fue suspendida definitivamente y los relaves se depositan, actualmente, en el Embalse de Relaves de Quebrada Honda. En 2002, aprovechando al máximo el agua para cubrir por completo los relaves y dar formación a espejos de aguas con porciones que se encuentran cubiertas de vegetación, se dio por cumplida la remediación de la Reserva de Relaves de Ite. Actualmente, los humedales de Ite representan un ecosistema que cuenta con la más alta población de aves acuáticas.

Palabras clave: Bahía de Ite, contaminación, Humedales, minera.

\begin{abstract}
The present article aimed to tell the history of the pollution in Ite bay located in Tacna, it is an important case of pollution due to the increase of solids and the presence of heavy metals due to the activity of the Toquepala and Cuajone mines. Beginning its operations between 1960 and 1976, Ite wetlands are exposed to great pollution by the tailings that have been deposited in Ite bay deteriorating the sea coast, modifying the sea coast until reaching a total area of more than 1,600 hectares, with a kilometer and a half wide and 12 kilometers long, In December 1996, the tailings discharge was permanently suspended and the tailings are currently deposited inland at the Quebrada Honda Tailings Reservoir, In 2002, water was used to the maximum to completely cover the tailings forming water mirrors with parts that are covered with vegetation, the remediation of the Ite Tailings Reserve was completed, currently Ite wetlands represent an ecosystem that has the highest population of waterfowl.
\end{abstract}

Keywords: Ite bay, pollution, wetlands, mining.

${ }^{1}$ Master of Sciencie con mención en Gestión Ambiental y desarrollo Sostenible, Ingeniero Metalurgista. E-mail:

Alex_212_y@hotmail.com

${ }^{2}$ Ingeniero ambiental, ingeniero civil. E-mail: Wachc300@gmail.com

Presentado: 05/10/18, Aprobado: 25/11/19 


\section{INTRODUCCIÓN}

En el hemisferio sur, la evidencia de contaminación en operaciones mineras durante el Imperio Inca (AD 1438-1532) tuvo un impacto insignificante en el ambiente sudamericano (Uglietti, Cooke, Gabrielli, Vallelonga, \& Thompson, 2015).

Los incas tuvieron una fuerte presencia en el litoral de Ite, no solo en el aprovechamiento de los recursos de lomas, sino también en la recolección de los recursos marinos. De esta época existen, además, áreas de petroglifos que destacan por su carácter iconográfico, con una ubicación estratégica en los caminos (Municipalidad de Ite, 2019).

Para el Perú, con sus 3080 kilómetros de litoral, la zona costera posee una singular importancia. Sin embargo, en la actualidad, las bahías, estuarios y lagunas costeras se han visto sometida a una fuerte presión por contaminación humana e industrial, especialmente en las últimas cuatro décadas, lo que ha afectado seriamente a la integridad del ecosistema marino(Ibárcena, 2011).

En el Perú, el subsector minero, esa actividad extractiva que proveía de la materia prima para el desarrollo (Castro, 2015), aparece reiteradamente como el sector industrial más contaminante de cuerpos de aguas superficiales y subterráneas, especialmente con cargas contaminantes de metales pesados entre otros contaminantes descargados al ambiente (Balvín \& Amezaga, 2006). Dichos impactos se han visto multiplicados desde que la tecnología moderna permite la explotación de minas a gran escala con baja ley de mineral (Balvin \& Amezaga, 2006) (Miguel, Prado, Día, \& Mosta, 2013).

En Ite, estaban orgullosos de sus peces extraídos de un mar limpio y bravío y un río que tenía agua casi todo el año y que traía hermosos camarones y lisas. Se consumían productos del mar, por ejemplo, mariscos, corvinas y lenguados como en pocos lugares del país. Pescaban en las laderas cerca de Ite y en verano disfrutaban de sus limpias, hermosas y libres playas(Lora, 2005).
El 12 de diciembre de 1952, Southern Perú Copper Corporation (SPCC) fue constituida en el estado de Delaware, Estados Unidos (Southern Copper, 2013) (Southern Copper, n.d.-a). Posteriormente, se estableció una sucursal por escritura pública otorgada ante el Notario Público de Lima, Dr. Ricardo Fernandini Arana, el 6 de noviembre de 1954 (Southern Copper, 2013) (Court, O'Brien, Ayala, Corcega, \& Enciso, 2013) (Southern Copper, 2017) (Southern Copper, 2016), y se estableció en Perú, la cual suscribió un convenio bilateral con el gobierno peruano para el desarrollo y explotación de la mina Toquepala (Southern Copper, n.d.-a) (Municipalidad Provincial de Tacna, 2014) (Southern Copper, 2017).

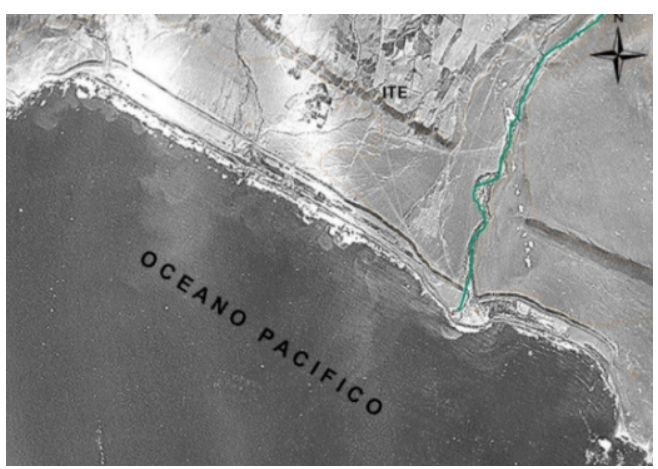

Figura 1: Bahía de Ite antes de la explotación minera 1955.

Fuente:Oviedo (2014)

En 1956, los trabajos para el desarrollo de la mina de Toquepala en Tacna, Perú, comenzaron sus actividades (Southern Copper, n.d.-a). Posteriormente, entran en operación las minas de Toquepala y Cuajone, que iniciaron en 1960 y 1976, respectivamente (Southern Copper, 2017) (Southern Copper, 2016) (Southern Copper, n.d.-a).

Los principales productos metálicos de Southern fueron el cobre, molibdeno y la plata (Court et al., 2013). Como consecuencia de las operaciones, las aguas del río se convirtieron en medio de transporte de los relaves hasta las canchas y luego arrojadas al Océano Pacífico por el río Locumba y la bahía de Ite, terminando contaminadas por metales pesados (FOCO, 2008).

El 26 de abril de 1961 se dio la creación política del distrito de Ite, mediante Ley $\mathrm{N}^{\mathrm{o}} 13660$, 
promulgada posteriormente con fecha 12 de junio del mismo año, por el Presidente Constitucional de la República Don Manuel Prado Ugarteche y el Ministro Ricardo Elías Aparicio (Municipalidad de Ite, 2019). "ITE" no es un término etimológico ni mucho menos un nombre quechua, son las siglas de "Irrigación de Terrenos Eriazos", la pujante acción de agricultores encabezados por arequipeños a mediados del siglo pasado, convirtieron en un gigantesco oasis los desiertos al noroeste de Tacna; tierra adentro de la angosta y reseca bahía (Vizcarra, 2013) (Vizcarra, 2008).

El 19 de diciembre de 1969, se suscribió el contrato Cuajone entre el Gobierno Revolucionario de las FFAA y la SPCC; aplicándose el mismo Código de Minería de 1950. En él quedaba en el olvido todo delito anterior y además se le reconocía a la SPCC inversiones previas, estabilidad tributaria, tratamiento financiero preferencial, libre comercialización del cobre, otorgamiento de garantías (como libre disponibilidad de divisas), fuentes de agua y energía seguras, y permiso para ampliar el complejo minero (Lora, 2005).

En 1972, tras contrastar con observaciones realizadas en 1960 por el Jefe de División de Investigación de Pesquería y Caza del Ministerio de Agricultura que mencionaba la existencia en la zona de una densa población de crustáceos y una fauna y flora algológica normal en este hábitat, la Cooperativa Agraria de Ite elevó al Ministerio de Pesquería un Memorial denunciando los daños a los recursos ictiológicos de la zona causada por la descarga de los relaves al río Locumba, al evidenciarse la desaparición de especies como el camarón, la lisa, erizos y almejas(FOCO, 2008).

En setiembre de 1975, fue elaborado el Proyecto de Almacenamiento de Relaves de Toquepala, Cuajone y Quellaveco por los Consultores Técnicos Asociados S.A. En 1977, se realizó el Estudio de Contaminación el Río Locumba y Playa de Ite. La Comisión Multisectorial R.M. 0044-77, en 1978, presentó el Informe "Mailing Disposal - Cerro Morrito Depresion” S. M. Tarbutt SPCC 1978.
En 1979, se presentó el Informe "Tailing Disposal at Ite Natural Basin" F. M. Stevenson SPCC, 1979. En diciembre de 1984, se da el Dictamen $N^{\circ} 2516 / 84-S$, elaborado por la Comisión Investigadora del Senado (FOCO, 2008).

La provincia de Jorge Basadre fue creada por Ley $\mathrm{N}^{\circ} 24799$ del 21 de abril de 1988, abarcando los distritos de Locumba, Ite e Ilabaya(Rodríguez \& Carrasco, 2007).

En 1989, fue aprobado por D.S. $\mathrm{N}^{\circ} 020-89$ PCM que, los relaves no debían continuar descargándose a la orilla del mar, por graves problemas de contaminación (FOCO, 2008). Solo aprovechaban el $1 \%$ del total de mineral que extraían, por lo que el $99 \%$ de los relaves eran descargados utilizando $21 \mathrm{~km}$ (OCMAL, 2015) del río Locumba, antes de su desembocadura en el mar (Balvín, 1995) (Balvín \& Amezaga, 2006) (Wiesse, 2015) (Miguel et al., 2013). Del $30 \%$ al $40 \%$ estaban constituidos por sólidos gruesos de la fracción más gruesa, por acción de las olas son devueltos a la playa ocasionando cambios geomorfológicos(Pilco \& Diaz, 2005)(Balvín, 1995). Esta acción de las olas y las corrientes sobre los sólidos formaron una playa de $10 \mathrm{~km}$ de largo que crecía a un ritmo de 40 a $60 \mathrm{~m}$ por año; y mar adentro, los relaves de menor granulometría y más pequeños precipitaban en el fondo marino, sepultando los sedimentos marinos ricos en materia orgánica, empobreciendo las aguas (Balvín \& Amezaga, 2006). Esta reserva contiene el $40 \%$ del total del relave descargado en el mar. La acumulación de las arenas causó que la playa fuera creciendo, ganando terreno al mar. Debido a la dirección de las corrientes marinas y a los fenómenos de afloramiento fueron formando la Reserva de Relaves de Ite (RRI) (Southern Copper, n.d.-b)(Balvín, 1995).

Estudios realizados en 1991 en la zona revelaron la presencia de altas concentraciones de arsénico, cadmio, plomo, cobre, cromo y cianuro con valores que superan los límites establecidos en la Ley de Aguas del Perú para zonas de pesca de mariscos y bivalvos. El alto contenido de metales pesados en la bahía provocó la contaminación de mariscos y peces 
(pelágicos y bentónicos), su desaparición por la elevada toxicidad o la bioacumulación. Asimismo, el aumento de la turbidez provocó la desaparición de algunas especies (Balvín \& Amezaga, 2006).

\section{Soluciones que se buscaron}

Primera: Esta opción consistía en transportar los relaves por gravedad, mediante tubería de polietileno de alta densidad hasta Punta San Pablo (ubicada a $15 \mathrm{~km}$ al sur de la Bahía de Ite) donde se entregarían a un tanque mezclador de concreto, para luego ser descargados por gravedad al mar mediante un emisor submarino de $170 \mathrm{~m}$ de longitud y a una profundidad de 12 a $30 \mathrm{~m}$. El costo de operación anual se estimó en 500000 dólares, por RESCAN ENVIRONMENTAL CONSULTANTS INC de Canadá. Se consideró como la mejor solución a nivel técnico, económico y ambiental, sin considerar sus numerosos aspectos negativos a saber(FOCO, 2008).

Segunda: La opción ganadora fue construir la presa en la zona de Quebrada Honda. En primer lugar, porque era de fácil acceso y cercana a las operaciones de SPCC. El hecho de que se encontrara en una quebrada amplia y de pendiente relativamente suave permite almacenar el íntegro del volumen de relave detrás de un solo dique. Por último, al recuperar $1 \mathrm{~m}^{3} / \mathrm{seg}$ de agua, se logra ampliar la frontera agrícola, hasta en 2 mil hectáreas. Estos estudios fueron realizados por KLOHN CFONOFF Consulting Engineers y sus resultados fueron presentados al Ministerio de Energía y Minas del Perú en marzo de 1991 (FOCO, 2008).

El 17 de febrero de 1992, se realizó en la Sala del Tribunal Internacional del Agua en Ámsterdam, Holanda, un "Juicio" a las actividades de la SPCC. En la misma, participaron la doctora Doris Balbín, el biólogo Juan Tejedo, el alcalde de Ilo (por aquel entonces) Ernesto Becerra y la Contralora General de la República, doctora Luz Aurea Saenz, quien precisó claramente que su despacho había investigado a más de 75 funcionarios que habrían estado involucrados en actos de corrupción por no aplicar las leyes peruanas durante más de 30 años. Sin embargo, la empresa no asistió a realizar su descargo, afirmando que el Tribunal Internacional del Agua no estaba reconocido por las Naciones Unidas. Además, cuestionó el accionar de la entidad denunciante ONG LABOR. Pese a contar con experimentados profesionales en el campo bio-ecológico, la ONG fue desacreditada a nivel profesional y científico. Por otro lado, Southern Copper argumentó que sus acciones en cuanto a la disposición de los relaves y el uso de fuentes hídricas contaron con la autorización y licencias respectivas proporcionadas por el Estado Peruano (FOCO, 2008).

De los relaves depositados en el mar, SPCC manifestó que era imposible almacenarlos en tierra por razones de espacio y características geográficas. Por eso, debían arrojarlos al mar, ya que por otro lado "no causarían ningún daño a la ecología marina”(FOCO, 2008).

Uno de los más importantes informes fue el realizado hacia 1992, por la Organización LABOR, el estudio titulado "Contaminación por metales pesados tóxicos producidos por las operaciones minero-metalúrgicas de la SPCC", llegando a varios descubrimientos. El $55 \%$ de estos relaves tenían contenido sólido. Los principales elementos y sustancias contenidos en los relaves eran cobre, plomo, mercurio, zinc, fierro, sílice, cianuro, grasas, etc. Aproximadamente unos $300 \mathrm{~km}^{2}$ de la Bahía de Ite quedaron contaminados (Lloja, Carbajal, \& Hurtado, 2008)(FOCO, 2008).

En 1994, como parte del acuerdo de bases firmado entre el gobierno peruano y SPCC, se construyó el envase de relaves de quebrada honda (ERQH). Se iniciaron trabajos de pruebas para vegetar algunas áreas del depósito de relaves (Oviedo, 2014). El dique de contención de Quebrada Honda se inauguró en 1995. Mientras tanto, los relaves, anteriormente arrojados al mar, ya suman miles de millones de toneladas, poniendo en riesgo a los pastores del área que utilizan los pastos naturales con los que alimentan a sus ganados, cuyos productos comercializan en el mercado regional (FOCO, 2008). Más tarde, en octubre de 1995, la empresa fue renombrado como Southern 
Copper Corporation (SCC) (Southern Copper, 2013)(Southern Copper, n.d.-a).

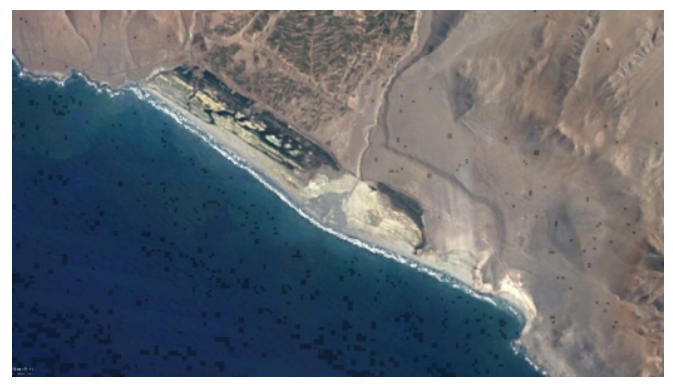

Figura 3: Bahía de Ite 12/30/1996.

Fuente: Google Earth pro (2019)

Desde el inicio de las operaciones de Toquepala y Cuajone hasta diciembre de 1996, las concentradoras descargaban un total de 785 millones de toneladas de relaves/jales (Southern Copper, n.d.-b), la impunidad duró hasta 1996, cuando construyeron un embalse de relaves(OCMAL, 2015).

Así, en diciembre de 1996, de acuerdo con el programa de adecuación y manejo ambiental (PAMA), la descarga de relaves fue suspendida definitivamente y desde esa fecha, los relaves de las plantas concentradoras de Toquepala y Cuajone se depositan tierra dentro en Quebrada Honda (Oviedo, 2014). Sin embargo, la playa había crecido hasta alcanzar un área total de más de 1600 hectáreas, con un kilómetro y medio de ancho y 12 kilómetros de largo (Southern Copper, n.d.-b), provocando una variación de la línea de costa(Gonzales, 2012), situada a $45 \mathrm{~km}$ al sureste de Ilo y al noreste de la ciudad de Tacna(OEFA, 2013).

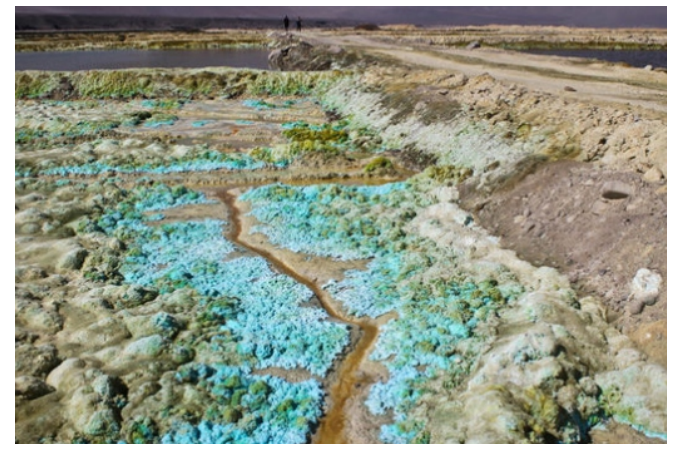

Figura 2: Algunas áreas sin remediar.

Fuente: Fotografía de Pajuelo (2015) en Diario La República
La emisión de relaves también dejó consecuencias socio-económicas:

- La zona litoral (Playa Inglesa y Caleta Meca Grande) que se dedicaba a la pesca y recolección de los recursos hidrobiológicos quedó despoblada.

- Poblaciones como Ite y Locumba vieron limitadas sus actividades recreacionales y deportivas como los deportes acuáticos.

- El establecimiento de industrias procesadoras de recursos hidrobiológicos quedó postergado.

- La ausencia de la población económicamente activa que vivía única y exclusivamente de la pesca y la recolección desfavorece la producción.

- Fue cancelada la ejecución del Proyecto Complejo Pesquero Punta Meca Grande y Puerto Grau (Caleta Morro) afectando la economía regional(FOCO, 2008).

Southern Perú descargo los relaves de sus minas Toquepala y Cuajone durante 36 años contaminando la bahía. Haciendo uso del río (FOCO, 2008) (Balvín \& Amezaga, 2006) (Miguel et al., 2013)(Southern Copper, n.d.-b).

En 1997, se aprueba el PAMA de SPCC, se inician los trabajos de vegetación y remediación ambiental de la playa de relaves, habiéndose efectuado primeramente ensayos para determinar la mejor estrategia para la remediación ambiental, la que debería ser eficiente, económica, y sostenible (Oviedo, 2014), como parte del compromiso del Programa de Adecuación y Manejo Ambiental (PAMA) que SPCC suscribió con el Gobierno Peruano(Southern Copper, n.d.-b), con un plazo de ejecución de 10 años calendario, repartida en 36 actividades de mitigación (Comisión de Ambiente y Ecología, 2004).

Para evitar la formación de las vetas de color rojizo y verde, producidas por la oxidación de los minerales presente en los relaves con el oxígeno atmosférico, fue necesario cubrir el relave con agua, pues la escasa proporción de oxígeno evita la oxidación. Para esto, se tuvo que aprovechar al máximo el agua (Vizcarra, 2008)(Vizcarra, 2013) cubriendo por completo los relaves y dando formación a lagunas 
superficiales (Lloja et al., 2008) (Vizcarra, 2013)(Pascó, 2000).

Uno de los aspectos más importantes de la polución es la representación de la materia inorgánica antrópica, la cual es considerada como esencial y tóxica dependiendo tanto de la especiación química del elemento (Ibárcena, 2011).

En noviembre de 1999, el Grupo México S.A.B. de C. V., una sociedad constituida de acuerdo a las leyes de la República de México, adquirió en los EE.UU. el $100 \%$ de las acciones de ASARCO lncorporated, que hasta ese momento era el principal accionista de Southern Peru Copper Corporation. De esta manera, SPCC se convirtió en una subsidiaria de Grupo México(Court et al., 2013).

En 2002, el Gobierno Peruano dio por cumplido el compromiso de remediación de la Reserva de Relaves de Ite que SPCC había contemplado en el PAMA (Southern Copper, n.d.-b). Con una superficie de 1600 hectáreas, este exitoso programa de remoción de contaminantes se ha traducido en el humedal más extenso y de mayor diversidad de aves acuáticas en la costa del país; y se ha convertido, también, en un foco de atracción turística que favorece el desarrollo económico (Southern Copper, 2017) (Southern Copper, 2016).

Se continúa con actividades de mantenimiento en dicha zona, especialmente en el control y manejo del agua para garantizar la estabilidad a largo plazo del humedal (Gonzales, 2012) (Southern Copper, n.d.-b). La permanencia del humedal ha creado un ecosistema muy biodiverso, debido a la abundancia de alimento y a la variedad de zonas de vida que existen en la Reserva de Relaves de Ite, en la cual se encuentran especies originarias como la grama salada y la verdolaga y, posteriormente, junco y totora (Southern Copper, n.d.-b). Es así que se da un ecosistema con especies propias del lugar, como distribución espacial de las especies de la vegetación (Municipalidad Provincial de Jorge Basadre, 2019). La Grindelia glutinosa, Lippia nodiflora, Ambrosia peruviana, Cenchrus echinatus
Cynodon dactylon, Bacopa monieri, Scirpus californicus, Thypha dominguensis, Paspalum vaginatum son de tipo contagiosa (Lloja et al., 2008).

El excedente de agua decantada del Embalse de Relaves de Quebrada Honda es enviado superficialmente aguas abajo para su reutilización en fines de protección ambiental minera en la Reserva de Relaves de Ite, de acuerdo a lo contemplado en la Resolución Directoral N 178-94-EM/DGM(OEFA, 2013) (Ibárcena, 2011)(Ibarcena, 2012)(Pascó, 2000) y se cuenta con la autorización de vertimiento, por la Autoridad Nacional del Agua mediante Resolución Directoral N 044-2010-ANADGCRH, de fecha 15 de septiembre de 2010 (OEFA, 2013) (Walsh Peru S.A., 2013) (Walsh Peru S.A., 2011). Dicha situación genera extensiones de marismas, pantanos, turberas o aguas de régimen natural o artificial, permanentes o temporales, estancadas o corrientes, dulces, salobres o saladas (Vizcarra, 2008) (Lloja et al., 2008). Formadas por un grupo de lagunas de agua con una profundidad máxima de 2 m (OEFA, 2013) (Tabilo, Burmeister, Chávez, \& Zöckler, 2016), incluyendo las extensiones de agua marina cuya profundidad en marea baja no exceden de los seis metros y cubren la bahía de Ite. (Vizcarra, 2008) (Lloja et al., 2008). El acceso a la zona está prohibido (Epensa, 2014) (Pajuelo, 2015), ya que los humedales serían de propiedad de la empresa minera Southern Perú (Radio Uno, 2010). Actualmente, los Humedales de Ite representan un ecosistema de altísimo valor natural, considerado incluso dentro de una categoría de reconocimiento internacional (IBA - "Important Bird Area"), y que a nivel nacional es uno de los más grandes humedales de la costa peruana que cuenta con la más alta población de aves acuáticas registrada durante los Censos Neotropicales de Aves Acuáticas (2004 y 2005). Esto los convierte en un recurso natural que requiere ser manejado para asegurar su sostenibilidad y al mismo tiempo generar un beneficio para las comunidades locales. De tal forma que, estas tomen conciencia y participen activamente en el proceso de conservación (Vizcarra, 2008) (Vizcarra, 2013) (Vizcarra, 2010). 
Siendo una de estas actividades el turismo practicado de una forma sustentable, el cual es un factor multiplicador de oportunidades de participación que permitirían tener un doble beneficio; generar una fuente de ingreso para las comunidades locales, quienes pueden participar en los diferentes momentos de prestación de servicios en la cadenaee turística (guianza, operación, alimentación, transporte, etc.) (Vizcarra, 2013)(Vizcarra, 2008).

En 2009, incrementarían a un total de 139 especies de aves observadas en los Humedales de Ite hasta la fecha, lo que evidencia la importancia de estos humedales para albergar a estas especies, algunas consideradas amenazadas en la legislación nacional (Vizcarra, Hidalgo, \& Chino, 2009).

Un impacto que no tuvo precedentes en toda la historia humana en su época (Uglietti et al., 2015) se trasformó en los humedales de la costa peruana que hoy constituyen un "punto de paso" para numerosas especies de aves migratorias, formando el Corredor Biológico que va desde los Manglares de Tumbes hasta los humedales de Ite en Tacna, pasando por Paraíso, Medio Mundo, Puerto Viejo entre otros (Sanchez, Blas, \& Fernandez, 2010). sirve de refugio para aves (Pilco \& Diaz, 2005) y el segundo más grande de Sudamérica(Peru.com, 2019) se consideran dentro de la clasificación de humedales naturales porque no han sido creados intencionalmente por el hombre (Moschella, 2012).

Respecto a las aves en el 2013, estas predominan debido a la presencia de los humedales, ya que albergan a especies endémicas, especies migratorias, especies emblemáticas y especies residentes. Las aves en su totalidad están representadas por 44 familias, 106 géneros y 153 especies (Municipalidad de Ite, 2013)(Peru.com, 2019).

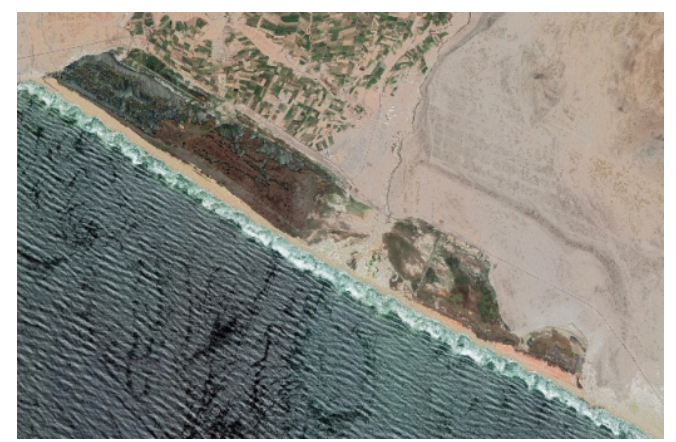

Figura 4: Bahía de Ite 07/30/2019

Fuente:Google Earth Pro (2019)

\section{REFERENCIAS}

Balvín, D. (1995). Agua, minería y contaminación. El caso Southern Perú. LABOR, Ilo, Perú. Recuperado de: http://www.labor.org.pe/wpcontent/uploads/2014/10/AGUAMINERIA-Y-CONTAMINACIONEL-CASO-SOUTHERN-PERU2018.pdf

Balvin, D., \& Amezaga, J. (2006). Agua , Minería y Cuenca Balance y Propuesta de Agenda. EMIRSA, Lima, Peru. Recuperado de: http://www.labor.org.pe/webermisa/i mages/ERMISAD1.pdf

Balvín, D., \& Amezaga, J. (2006). Estado de la Situación sobre la Gestión del agua en la Minería: El caso Peruano. Ermisa, Lima, Peru. Recuperado de: http://www.labor.org.pe/webermisa/i mages/ERMISAD1.pdf

Castro, D. (2015). Contaminación minera en Perú se inició durante la época colonial. Diario El Comercio, Publicado 02 nobiembre 2015. $\mathrm{R} \mathrm{e} \mathrm{c} \mathrm{u} \mathrm{p} \mathrm{e} \mathrm{r} \mathrm{a} \mathrm{d} \mathrm{o} \mathrm{d} \mathrm{e} \mathrm{:}$ https://elcomercio.pe/blog/expresion genetica/2015/02/contaminacionminera-durante-epoca-colonial 
Comision de Ambiente y Ecologia. (2004). Informe Final de la Subcomisión Investigadora del cumplimiento de los PAMA' $\mathrm{s}$ y EIA' $\mathrm{s}$ de las Empresas Mineras. Comgreso de La Republica. Recuperado de: http://www2.congreso.gob.pe/Sicr/C omisiones/2004/Ambiente 2004.nsf/ 4Documentosweb/D5D309554773D1 26805256F470079E1B3/\$FILE/INF ORME FINAL DE LA SUB CO MISION.PDF

Court, E., O'Brien, J., Ayala, C., Corcega, Y., \& Enciso, H. (2013). Reporte Financieros CENTRUM Southern Peru Copper Corporation, sucursal del Peru. Centrum, Universidad Católica Del Perú. Recuperado de: https://www.latinburkenroad.com/do cs/BRLA Southern Peru Copper (201303 Spanish).pdf

Epensa. (2014). Presentan observaciones en los humedales de Ite 28-03-2019. Diario Correo, Publicado 23 octubre 2014 . R e c u p e r a d o d e : https://diariocorreo.pe/peru/presentan -observaciones-en-los-humedales-deite-337333/

Foro Ciudadano del participacion por la justicia y los derechos humanos FOCO. (2008). Informe especial sobre las operaciones de Southern Copper Corporation en el sur de Perú. Buenos Aires, Argentina. Recuperado de: http://www.inpade.org.ar/oetdocumentacion y base de datos/oetinformes/Southern Copper Corporation en el Peru.pdf

Gonzales, E. G. (2012). Foro : Agua y Biodiversidad Necesidades de agua en humedales costeros. MINAM, San Isidro, Peru. Recuperado de: http://www.minam.gob.pe/diadiversi d a d / w p content/uploads/sites/63/2015/01/pre sentacion4.pdf

Google Earth pro. (2019). Imagenes Historicas - Ite 12/30/1996. 07/30/2019.

Ibarcena, L. (2012). Incidencia de la Contaminación por Metales Ecotóxicos en la Fauna Marina Bentónica en la Bahía de Ite, Provincia de Jorge Basadre
Grohmann de Tacna. Rev. Ciencia \& Desarrollo, 14, 2834.

Ibárcena, L. W. (2011). Estudio de la Contaminación por Metales Ecotóxicos en Sedimentos en la Bahía de Ite, Tacna. Rev. Ciencia \& Desarrollo, 13, 2835.

Lloja, L., Carbajal, V., \& Hurtado, H. (2008). Evaluación de la Distribución Espacial de la Flora Silvestre de los Humedales de Ite, Provincia Jorge Basadre Grohmann 2008. Rev. Ciencia Y Desarrollo, (12), 8792.

Lora, J. (2005). Transnacionales mineras y ecocidio en el Perú. El Grupo México y su política neoliberal. Rev. Quivera, 7(1), 316337.

Miguel, J., Prado, F., Día, F. V., \& Mosta, E. T. (2013). Agua, minería y comunidades campesinas en la región Tacna. Rve. Ciencia \& Desarrollo, (15), 7380 .

Moschella, P. (2012). Variacion y Proteccion de Humedales Costeros frente a procesos de Urbanizacion: casos Ventanilla y Puerto viejo. Tesis Maestria de PUCP, Lima, Perú.

Municipalidad de Ite. (2013). Resumen de los ejes o Estudios temáticos para la elaboración de la microzonificación Ecológica económica del distrito de Ite.

Municipalidad de Ite. (2019). Historia. $\mathrm{R} \mathrm{e} \mathrm{c} \mathrm{u} \mathrm{p} \mathrm{e} \mathrm{r} \mathrm{a} \mathrm{d} \mathrm{o} \mathrm{d} \mathrm{e} \mathrm{:}$ http://www.muniite.gob.pe/web/distri to/historia

Municipalidad Provincial de Jorge Basadre. (2019). Recursos Naturales. Publicado 22 marzo 2019. $\mathrm{R} \mathrm{e} \mathrm{c} \mathrm{u} \mathrm{p} \mathrm{e} \mathrm{r} \mathrm{a} \mathrm{d} \mathrm{o} \mathrm{d} \mathrm{e} \mathrm{:}$ http://www.munijorgebasadre.gob.pe /pagina/web inst/prov recursos.php

Municipalidad Provincial de Tacna. (2014) Plan de desarrollo concertado provincial de Tacna 2011-2021 ( Actualizado Marzo 2014 ). $\mathrm{R} \mathrm{e} \mathrm{c} \mathrm{u} \mathrm{p} \mathrm{e} \mathrm{r} \mathrm{a} \mathrm{d} \mathrm{o} \mathrm{d} \mathrm{e} \mathrm{:}$ https://www.munitacna.gob.pe/pdfs/p dc2011.pdf

Observatorio de Conflictos Mineros de América Latina OCMAL. (2015). Southern Copper dice que cuida el medio ambiente pero informes 
oficiales señalan lo contrario. Publicado 3 julio 2015. Recuperado de: https://www.ocmal.org/southerncopper-dice-que-cuida-el-medioambiente-pero- informes-oficialessenalan-lo-contrario/

OEFA. (2013). Resolución Directoral N5822013-OEFA-DFSA/ Expediente $\mathrm{N}^{\circ}$ 162-2012-DFSAI/PAS. Recuperado d e : https://www.oefa.gob.pe/?wpfb $\mathrm{dl}=6$ 720Oviedo, D. (2014). Medidas de mitigación ambiental en la bahia de ITE Programa de adecuación y manejo ambiental. Primer Congreso Internacional de Turismo Ornitológico Ite.

Pajuelo, R. (2015). Publicidad de minera Southern no dice toda la verdad sobre los humedales de Ite. Diario La República, Publicado 26 septiembre 2015 . R e c u perad o d e : https:// arepublica.pe/enportada/884236-publicidad-deminera-southern-no-dice-toda-laverdad-sobre-los-humedales-de-ite

Pascó, A. (2000). El impacto del programa de estabilización y las reformas estructurales sobre el desempeño ambiental de la minería de cobre en el Perú: 1990-1997.

Peru.com. (2019). Tacna: Despierta tus sentidos en los Humedales de Ite (FOTOS). Peru.com, Publicado 30 junio 2015. Recuperado de: https://peru.com/viajes/conozcaperu/tacna-despierta-tus-sentidoshumedales-ite-fotos-noticia- 371873

Pilco, P., \& Diaz, J. (2005). Visión Panoramica de las actividades mineras en el Perú 2005. Talleres Gráficos de SINCO Editores, Breña, Perú.

Radio Uno. (2010). Conservación de los humedales de ITE genera contradicciones en el GRT. Radio Uno, Publicado 21 abril 2010. $\mathrm{R} \mathrm{e} \mathrm{c} \mathrm{u} \mathrm{p} \mathrm{e} \mathrm{r} \mathrm{a} \mathrm{d} \mathrm{o} \mathrm{d} \mathrm{e} \mathrm{:}$ http://www.radiouno.pe/noticias/147 98/conservacion-humedales-itegenera-contradicciones-grt

Rodríguez, H., \& Carrasco, R. (2007). El aji en Ite (Tacna). Recuperado de: https://iadiperu.jimdo.com/app/down
load/10801584660/Humberto+Rodrí guez+Pastor+y+Rebeca+Carrasco $+\mathrm{A}$ tachao.pdf? $\mathrm{t}=1469480630 \&$ mobile $=$ 1

Sanchez, G., Blas, N., \& Fernandez, G. (2010). Informe nacional sobre el estado del ambiente marino del perú. Instituto Del Mar Del Peru, Callao, Peru.

Southern Copper. (n.d.-a). Historia Southern Perú Copper Corporation SPCC. $\mathrm{R} \mathrm{e} \mathrm{c} \mathrm{u} \mathrm{p} \mathrm{e} \mathrm{r} \mathrm{a} \mathrm{d} \mathrm{o} \mathrm{d} \mathrm{e} \mathrm{:}$ http://www.southernperu.com/ESP/a cerca/Pages/PGHistoria.aspx

Southern Copper. (n.d.-b). La remediación de Ite, reserva de relaves. Recuperado d

http://www.southerncoppercorporatio n.com/ESP/dessos/CasosEstudio/Pag es/PGCasoEstudio ITE.aspxSouther n Copper. (2013). Informe anual 2013 . R e c u p e r a d o d e : http://www.southernperu.com/esp/rel inv/2013/AnnualReport/m2013es.pdf

Southern Copper. (2016). Informe anual 2016. $\mathrm{R} \mathrm{e} \mathrm{c} \mathrm{u} \mathrm{p} \mathrm{e} \mathrm{r} \mathrm{a} \mathrm{d} \mathrm{o} \mathrm{d} \mathrm{e} \mathrm{:}$ http://www.southernperu.com/esp/rel inv/2016/AnnualReport/m2016e.pdf

Southern Copper. (2017). Informe anual 2017. $\mathrm{R} \mathrm{e} \mathrm{c} \mathrm{u} \mathrm{p} \mathrm{e} \mathrm{r} \mathrm{a} \mathrm{d} \mathrm{o} \mathrm{d} \mathrm{e} \mathrm{:}$ http://www.southernperu.com/ESP/re linv/2017/AnnualReport/m2017esp.p df

Tabilo, E., Burmeister, J., Chávez, C., \& Zöckler, C. (2016). Humedales y aves migratorias en la costa árida del Pacifico Sudamericano.

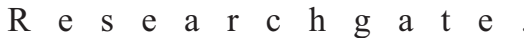
https://doi.org/10.13140/RG.2.1.300 4.4565

Uglietti, C., Cooke, C. A., Gabrielli, P., Vallelonga, P., \& Thompson, L. G. (2015). Widespread pollution of the South American atmosphere predates the industrial revolution by $240 \mathrm{y}$. Proceedings of the National Academy of Sciences, 112(8), 23492354.

Vizcarra, J. (2008). Los Humedales De Ite: Un Potencial Ecoturistico. OGD TUR TACNA., Tacna, Peru. Recuperado d http://www.turismotacna.com/LIBR O_HUMEDALES_DE_ITE_OGDT 


\section{URTACNA.pdf}

Vizcarra, J. (2013). Humedales de Ite: Un Potencial Ecoturístico. Turismotacna.com. Recuperado de: http://www.turismotacna.com/index. php?option $=$ com content\&view $=$ art icle\&id=196\&Itemid=137\&showall $=1$

Vizcarra, J. K. (2010). Nuevos registros ornitológicos en los humedales de Ite y alrededores, Tacna, Perú. Rev. The Biologist, 8(1), 120.

Vizcarra, J. K., Hidalgo, N., \& Chino, E. (2009). Adiciones a la avifauna de los Humedales de Ite, costa sur de Perú. Rev. Peruana de Biología, 16(2), 221225.

Walsh Peru S.A. (2011). Resumen ejecutivo estudio de impacto ambiental "ampliación de la concentradora Toquepala y recrecimiento del embalse de relaves de Quebrada Honda."

Walsh Peru S.A. (2013). EIA Ampliación de la Concentradora Toquepala y Recrecimiento del Embalse de Relaves de Quebrada Honda, Descripción Del Proyecto.

Wiesse, P. (2015). Contranatura. La historia de Southern en Perú. 60 años de destrucción medioambiental. Publicado 04 agosto 2015. $\mathrm{R} \mathrm{e} \mathrm{c} \mathrm{u} \mathrm{p} \mathrm{e} \mathrm{r} \mathrm{a} \mathrm{d} \mathrm{o} \mathrm{d} \mathrm{e} \mathrm{:}$ http://cosal.es/contranatura-lahistoria-de-southern-en-peru-60a n o s - d e - d e s t r u c c i o n medioambiental/ 